\title{
La acentuación en el español como segunda lengua en Nueva York
}

\author{
The accentuation in Spanish as Second Language in New York \\ Eduardo Barros Grela \\ Facultad de Filología, Universidad de la Coruña, España \\ e-mail: ebarros@udc.es
}

Este artículo consiste en un análisis de las dificultades mostradas por una serie de alumnos de nivel elemental de español como segunda lengua en la Universidad de Nueva York para realizar con éxito ejercicios prácticos de acentuación. El artículo propone una hipótesis que intenta explicar los motivos de estas dificultades fundamentada en la idea -ya anticipada por Elaine Tarone- de que el origen de los problemas para colocar acentos gráficos del alumnado cuya L1 es el inglés radica no tanto en la falta de acierto a la hora de localizar la sílaba tónica de la palabra, sino en el propio mecanismo de identificación de las sílabas y en la problemática división de palabras en sílabas.

Palabras clave: interlenguaje, fosilización, división en sílabas, contacto de lenguas, lingüística aplicada, español como segunda lengua.

This article is an analysis of the difficulties shown by a series of elementary level students of Spanish as a second language at the University of New York to successfully complete exercises of word accentuation. The article proposes a hypothesis that attempts to explain the reasons for these difficulties based on the idea, already anticipated by Elaine Tarone, that the origin of the problems shown by students whose L1 is English to write accent marks on appropriate syllables is not so much the lack of success when locating the stressed syllable of the word, but it is in fact the very mechanism of identification of the problematic syllables and the action of segmenting words into syllables.

Key words: interlanguage, fossilization, syllable segmentation, code-switching, applied linguistics, Spanish as a second language.

"...the syllable structure of the interlanguage is often markedly different from that of the target language."

Elaine Tarone

\section{INTRODUCCIÓN}

Hay dos principales razones por las que se hace necesaria una recapitulación de lo que ha significado hasta la primera década del siglo XXI la inmersión metodológica 
en la estructuración silábica como causa de un proceso de interlenguaje: por un lado, se trata de un rasgo diferenciador a la hora de aplicar la acentuación gráfica del español; por otro, la propia diferencia interlingüística entre la lengua inglesa y la lengua española se considera, aplicada a este ámbito, como un problema de primer orden para la producción correcta de estructuras mayoritariamente fónicas en la L2 ("target language").

Conociendo la gran relevancia de la formación silábica en la adquisición de una segunda lengua, cabe preguntarse el por qué de una tan reducida bibliografía dando luz a este campo, dentro de una disciplina tan joven pero tan impulsiva y creciente como la lingüística aplicada. Se han hecho -en contraste- numerosos estudios basados en la morfosintaxis como ente aglutinante de las teorías de interlenguaje que copan la corriente mejor establecida dentro del propio campo. Sin embargo, un estudio que trate de penetrar en la problemática que conduce a la dificultad de la interpretación silábica desde un punto de vista fonológico se va a encontrar con la dificultad añadida de contar con muy poco material bibliográfico. Existen, en realidad, escasos tratados en torno a esta aproximación metodológica para explicar un fenómeno presente en la adquisición de una L2. Entre ellos, los que más se aproximan al enfoque con que se ha dotado este estudio, se encuentra "Syllable Structure in SLA", de C. Greenberg; "On Stress and Syllabification", de J. McCarthy, o "Some influences on the Syllable Structure of Interlanguage Phonology", de E. Tarone. Todas estas investigaciones basaron sus aproximaciones en cómo la organización silábica influye de una manera directa en el proceso de producción articulada oralmente dentro del campo de la adquisición (o aprendizaje) de una segunda lengua, algunas de ellas desde marcos teóricos menos aislados, como el artículo de Greenberg; otros con una definición interpretativa mucho más identificada con una determinada corriente de pensamiento aplicado, como el estudio de Tarone con respecto al interlenguaje.

Todas ellas son puntos de partida de excepción para hacer despegar el reconocimiento analítico que este trabajo representa. Su misión es abrir un campo de exploración para futuras inmersiones de investigación. Si hasta ahora se había tenido escasamente en cuenta cómo un desconocimiento de las reglas de estratificación silábica afectaba al aprendizaje de la acentuación gráfica (e incluso en la prosódica) de la lengua española en estudiantes que poseen un conocimiento no nativo de la misma, en este momento cabe plantearse si la metodología tradicional para este propósito es realmente útil o no. El proceso que se trata de aplicar con este objetivo en la mayoría de los textos utilizados consiste en una explicación de las reglas de acentuación siguiendo el modelo de enseñanza aplicable para los alumnos que tienen el español como lengua materna y que, a través de un proceso de adquisición de la misma, poseen una capacidad de conocimiento silábico que los estudiantes que no tienen una competencia lingüística nativa no pueden siquiera pretender tener. Esta teorización lingüística será el pilar donde este estudio se va a apoyar para conformar una hipótesis en proceso de construcción dentro del ámbito de la lingüística aplicada.

Antes de entrar más a fondo en esta problemática, es conveniente introducir una breve contextualización del modo y de la forma del planteamiento que nos ocupa. El lugar y el momento donde todas estas dudas y problemas han surgido tienen una relevancia considerable dentro del estudio que se está tratando, ya que se hace difícil establecer una diferencia clara entre lo que es adquisición y lo que es 
aprendizaje. El lugar es el estado de Nueva York, y el momento es el de una masiva migración de, ya no sólo individuos, sino enteras comunidades hispanófonas. La respuesta a en qué medida este factor puede afectar el aprendizaje de una serie de reglas gramaticales o lingüísticas es que, en muchos casos, el corpus que está siendo utilizado para la realización de este estudio puede presentar signos equívocos por la influencia sociológica de la lengua española en esta comunidad concreta. Se trata de una fusión de los dos términos que hemos estado tratando para referirnos a la manera de asimilar una nueva lengua: por una parte, estos alumnos están recibiendo clases de lengua con una aproximación eminentemente comunicativa, pero insertas en un círculo teórico obsoleto y no por indeseado inevitable. En segundo lugar, las condiciones sociopolíticas de la realidad que envuelve a estos alumnos les hace estar expuestos a un uso desde moderado hasta cotidiano de esa misma lengua. Se puede pues considerar este momento para amalgamar ambas maneras lingüísticas como una situación idílica para el exitoso aprendizaje y la apropiación de una funcionalidad siempre deseada. Es importante, no obstante, mantener presente esta exclusividad situacional mientras se toma en consideración este estudio, ya que es hasta cierto punto este rasgo lo que lo diferencia de las situaciones presentadas en los anteriores trabajos, donde se trataban casos de inmersión relativa o ralentizada de los estudiantes en la lengua y de la lengua en los estudiantes. Es, de la misma manera, una característica de suma importancia para comprender la (supra) motivación añadida de los alumnos en el uso de la lengua que están aprendiendo, que no se da con otras que no están tan presentes en su realidad cotidiana. Para tratar el problema del corte silábico y su correlato de tildación en español para hablantes de éste como su segunda lengua se requiere de una heterogeneidad manifiesta por parte de los sujetos que participan en el proyecto, ya que reducir el ámbito de este estudio a una sola categoría de hablantes podría dañar la rigurosidad del proyecto. El objetivo de este estudio es observar las dificultades presentadas por un grupo de alumnos de español como segunda lengua en el ámbito estadounidense, cuya demografía está en sí definida por la heterogeneidad de sus habitantes. El muestreo propuesto en la sección práctica de este análisis busca, consecuentemente, representar esa heterogeneidad étnica y cultural propia del aula universitaria estadounidense, y observar cuáles son las repercusiones lingüísticas de las diferentes L1 en el problema de acentuación gráfica a partir de la separación silábica que se plantea aquí. La actuación de este estudio debe, por lo tanto, hacerse eco de esa heterogeneidad étnica y cultural y observar los efectos de ésta en los alumnos de español como segunda lengua.

Para concluir esta introducción, es necesario recordar que el fin último de este estudio se basa en el intento de seguir trabajando en la continua y paulatina mejora de la calidad de enseñanza aplicada a una segunda lengua; en descubrir si existe un problema, en aislar los motivos que conducen a ese trauma, y en plantear una suerte de posibles soluciones que han de ser matizadas, variadas $y$, probablemente, cuestionadas por una futura crítica que siga esta línea de estudio. En lo directamente aplicado al tema que se trata, el objetivo último es conseguir abrir un camino hacia el hecho de incorporar una explicación seria de lo que significa la sílaba en español durante las primeras sesiones de clase y, desde luego, antes de la introducción a la metodología de acentuación del español. 


\section{MARCO TEÓRICO}

Una relativamente nueva disciplina lingüística como la que nos ocupa surgió del concepto más general de lingüística y como resultado de una necesidad metodológica que actuase directamente sobre la cuestión de cómo el lenguaje es adquirido, primero, y cómo una segunda lengua es aprendida en segundo lugar.

Como ya se ha sugerido con anterioridad en la introducción, existe un número importante de diferentes teorías que hacen referencia a la adquisición de una segunda lengua y la problemática que ello involucra. Existen similitudes y, obviamente, diferencias entre ellas, y de la misma manera que en ocasiones se contradicen las unas a las otras, en otros momentos coinciden plenamente en determinadas concepciones.

Por supuesto, una de las dialécticas más interesantes en las que se identifican todas estas corrientes teóricas es la que discute la interrelación entre la L1 y la L2, es decir, la lengua materna del estudiante y la lengua a la que está expuesto dentro del entorno de aprendizaje que la clase conlleva. A pesar de los avances metodológicos que esta divergencia retórica incluye en paradójica existencia, aún hoy sigue siendo éste un tema que no ha quedado ni mucho menos claro con toda la investigación succionada. Existen al menos cuatro aproximaciones que merecen ser tenidas en cuenta de acuerdo con el estudio que Vivian Cook llevó a cabo en referencia al aprendizaje de una segunda lengua, pero también y al mismo tiempo, a la enseñanza de una segunda lengua. El mismo proceso entendido desde las dos principales ópticas que intervienen en la interacción dentro de la atmósfera comunicativa y guiada.

Los primeros modelos teóricos con aplicación pragmática que esta lingüista introdujo en su resumen crítico fueron los que estaban directamente relacionados con el "knowledge", con el conocimiento. Por supuesto, aquí aparece la figura de quien representó una revolución en el ámbito lingüístico durante los años sesenta, y que se ha convertido en la figura más importante dentro de su campo en los últimos años: Noam Chomsky. Y con él, su aproximación ontológica al campo lingüístico, basada en la concepción del lenguaje como algo innato al ser humano, como una función cerebral que se implica en todos y cada uno de los seres humanos con capacidad cerebral intacta o no dañada. Según Chomsky, la capacidad de lenguaje es algo que es congénito al individuo en la forma de una "gramática universal", explicada a grandes rasgos y por lo tanto de forma inexacta, como que todo individuo posee una programación gramática en su mente que es común al resto de personas en el mundo. Es una activación de ese conocimiento gramatical lo que permite al individuo desarrollar una capacidad lingüística plasmada en un determinado lenguaje que va siendo adquirido a medida que la persona va interaccionando con el medio que le abraza. En esta explicación tangencial de lo que es el pensamiento lingüístico de Chomsky, hay que hacer mención al hecho de que él entiende que el aprendizaje de una segunda lengua tiene una forma de suceder en cierta medida semejante a la de la adquisición de la lengua materna, pero con algunas diferencias entre la que destaca la falta de contacto con la sociedad que habla a diario esa lengua en particular. Sería un estudio de interés, como ya se ha comentado de manera implícita anteriormente, entender cómo se amalgaman ambas formas de proceder en la sociedad neoyorquina sin llegar a entrar en dimensiones de bilingüismos y diglosias. Para explicar esta teoría, se ha recurrido a una estratificación cognitiva que implica principios, por un lado, y parámetros por el otro. En general, se considera el lenguaje como un mecano construido en la mente 
y a partir de parámetros. Es tarea del inconsciente crear, a partir de estos parámetros, los principios lingüísticos que un determinado lenguaje necesita. Para el aprendizaje de una segunda lengua, ya no es tanto que haya que modelar esos parámetros como que haya que re-modelarlos, ya que los estudiantes escuchan ejemplos de esa nueva lengua y tratan de incorporarlos a una remodificación de sus parámetros. Al considerar la gramática como algo ya intrínseco al ser humano, quienes siguen esta teoría aplican a la enseñanza de una segunda lengua la no necesidad de enfatizar ese aspecto durante los momentos de la clase, y consideran de mayor importancia dedicar tiempo a establecer nuevos significantes para referentes reales; ven una mayor necesidad de énfasis pedagógico en el vocabulario.

El segundo grupo de modelos que Cook estudia es el de procesos ("processing models"), que se colocan en una posición teórica completamente opuesta a la que postulan los seguidores de la gramática universal. Para ellos lo más importante, más allá de gramáticas o vocabularios, para un exitoso producto en el aprendizaje de una lengua es ante todo la comunicación. Este modelo se puede considerar mucho más pragmático y funcional que el propuesto por Chomsky por estar dirigido hacia una realidad mucho más cercana, hacia una utilización de lo aprendido de una manera mucho más directa.

El tercer grupo lo forman los modelos multi-componenciales, que son hasta cierto punto una consideración ecléctica de las dos corrientes anteriores (lenguaje como conocimiento y lenguaje como proceso). El modelo de competencia y control es particularmente interesante porque considera que el lenguaje posee estos dos rasgos; el primero, para estudiar cómo el alumno conceptualiza el lenguaje en la mente, y el segundo, para saber cómo el alumno controla y procesa ese conocimiento en situaciones reales. Esto es especialmente aplicable a la teoría de interlenguaje que tiene lugar en la problemática que se está tratando en este trabajo, porque los procedimientos inadecuadamente tomados de la L1 van a distorsionar de manera radical el reciente conocimiento de una $\mathrm{L} 2$, de acuerdo con los términos que utiliza Cook.

El cuarto y último grupo de modelos es el social. Más parecido al segundo modelo en cuanto a que da una gran importancia a enfatizar el rol de la sociedad en el proceso de aprendizaje de una segunda lengua. La sociolingüística toma aquí un carácter realmente importante. Quizá sea éste el modelo que más se acomoda a la situación de la realidad de los estudiantes en la escuela universitaria neoyorquina de Stony Brook, donde la presencia latina, y con ella del idioma español, es importante, de manera que existe un contacto real entre los estudiantes y la comunidad hablante de la "target language". Sería, sin embargo, un error hacer de este modelo una entidad excluyente, ya que se dan muchos otros procesos, además del social, en el momento del aprendizaje.

Finalmente, hay que hablar de la teoría en la que este estudio está basado, que es la teoría del interlenguaje y que será comentada con mayor profusión en la próxima sección:

[...] an intermediate system located somewhere between the learner's native language and the target language, but governed by its own unique and coherent internalized rule system that rarely becomes totally congruent with the system of the second language. (Omaggio, 229). 
Como se puede ver por lo expuesto a modo de resumen hasta ahora, existe una gran discrepancia en torno a la manera de entender cómo funcionan los procesos cognitivos en el momento de aprender una lengua, ya sea en la adquisición de la propia lengua materna como en el aprendizaje de una segunda lengua (o "target language"). Sirva, pues, esta irresuelta dialéctica para ilustrar en qué medida puede influir la presencia de la L1 en las mentes de los estudiantes que están tratando de dividir en sílabas una palabra en la L2, padeciendo un conflicto que responde a la propia y diferente idiosincrasia de cada lengua, o en el momento de acentuar palabras en la L2, es decir, aplicar una regla que no existe en la L1 y que, por lo tanto, no debería presentar ningún tipo de problema interlingüístico en la producción de L2, pero que, por estar su aprendizaje basado en formas equivocadas (desconocimiento de la diferencia entre dividir sílabas en español y hacerlo en inglés), se da una situación de absoluto fracaso de aprendizaje.

\section{MARCo DE REFERENCIA}

De la misma manera que muchos otros autores que han aplicado esta teoría del interlenguaje a sus estudios sobre los problemas para aprender una segunda lengua, A. Omaggio explora la concepción de interlenguaje para tratar de responder a la pregunta en torno a los procesos del aprendizaje lingüístico como fuentes de producción de errores tanto a nivel hablado como a nivel escrito. Tratando de hacer una fusión de estos dos ámbitos, este trabajo seguirá también la misma teoría como punto de referencia.

Quien más en profundidad ha trabajado este concepto de interlenguaje es Larry Selinker; en su libro Rediscovering Interlanguage propone cinco procesos diferentes: "language transfer, transfer of training, strategies of second language learning, strategies of second language communication, and overgeneralization of target language rules" (89). Por ejemplo, los estudiantes que cometen errores debido al primer caso, es decir, interferencia de la lengua materna, normalmente muestran esos errores en pronunciación, sintaxis o morfología, entre otros. Desde luego es un buen primer paso para entender los problemas de los estudiantes del nivel más básico de español (SPN 111), para superar los problemas que aquí se están planteando. Otros ejemplos son los problemas que puedan ser causados por un uso indebido de materiales dentro del aula, donde gran parte del proceso de aprendizaje tiene lugar. Si esos materiales son incompletos o hacen un uso equivocado de los parámetros o estructuras que tratan, los estudiantes reflejarán a su vez esos mismos errores.

Otro rasgo importante del interlenguaje es lo que se ha venido a llamar "fosilización". Describe una incongruencia entre el interlenguaje y la L2, una serie de rasgos que se convierten en permanentes por la influencia del interlenguaje en la "target language" y que no pueden ser reparados posteriormente. Se podría adivinar aquí una consecuencia del mal uso de la división silábica como error para acentuar posteriormente las palabras. Una intervención inmediata es necesaria para atajar posibles fosilizaciones de los estudiantes a la hora de reconocer los golpes de voz en español y su representación gráfica. Estos aspectos serán también revisados, aunque de manera más esquemática, en la consecución de este trabajo. 
Antes de entrar directamente con el tema que me ocupa, creo necesario presentar dos temas realmente importantes. Por un lado, considero que el problema del interlenguaje como forma de catalizar la marcha del aprendizaje se debe no sólo a cuestiones puramente internas del alumno. Existen muchas circunstancias exteriores que pueden de la misma manera influir en la aparición de este problema: cómo el profesor introduce las lecciones, la motivación, la situación de la clase y un largo etcétera. Por otro lado, no me atrevo a considerar un gran daño el hecho de que no se haya estudiado demasiado este problema del interlenguaje aplicado a la acentuación gráfica como última receptora del error, ya que se descubre en un momento de plena decadencia de la conciencia ortográfica en español. En una aproximación comunicativa a la enseñanza de L2, los acentos gráficos no tienen la relevancia que pueden tener en otros ámbitos. Considero, sin embargo, que no hemos de caer en esa dejadez ortográfica, y mucho menos con personas a quienes se les está introduciendo la lengua por primera vez. Que la decrepitud de la seriedad ortográfica es un reflejo de la realidad linguística es algo que no me atrevo a afirmar. Que en caso de que así sea, hay que luchar contra ello, es también un asunto problemático y, sobre todo, polémico. Pero no son las principales intenciones de este trabajo sobrepasar las fronteras del más puro sentido de interlenguaje aplicado a la sílaba y al acento español.

Emilio Alarcos Llorach (1994) hace un estudio muy interesante sobre la forma y condición de la sílaba en el idioma español. Intenta delimitar la conceptualización de sílaba en lo que afecta a la lengua española, y para ello hace uso de una serie de definiciones que pueden ser de gran utilidad para la finalidad de este trabajo. Por un lado, dice que "la sílaba es una unidad funcional en la secuencia fónica", y la desvincula de cualquier tipo de relación o carga semántica.

La sílaba -continúa- aunque como complejo fónico puede cumplir funciones distintivas, pertenece exclusivamente a la expresión. Dentro de la secuencia fónica, la sílaba es la combinación mínima y autónoma de fonemas. La sucesión de las sílabas está condicionada por la alternancia variable del rasgo que llamamos acento. (131)

Esta información que ha sido resumida de manera tan brillante por el autor español nos lleva a un camino de no retorno al plano de la expresión. Lo que está implicando este autor es que la división silábica que se está tratando en este estudio depende en todo caso de cómo la misma se produce a un nivel expresivo. En su definición, Alarcos deja entrever la relación íntima entre la propia condición de la sílaba y la existencia de un rasgo llamado acento como causa implícita de la catalogación de la sílaba. Sin embargo, al hablar de este concepto, el autor de Gramática de la Lengua Española establece una importante diferenciación que es de sumo interés para este trabajo. Afirma que es un error confundir la estructura silábica que aparece en cada uno de los marcos en que se está considerando; es decir, el de las relaciones dentro de la palabra aislada y el de las relaciones en secuencias unitarias de palabras. Confirmo mi especial interés en un argumento de este tipo porque esta diferenciación es algo que no sucede en la lengua inglesa, de acuerdo con Quirk. Según el autor de A Concise Grammar of the English Language, en lengua inglesa existe la diferenciación entre "stress", "rhythm" e "intonation", siendo el primero "the prominence with which one part of a word or a longer utterance is distinguished from other parts. Thus we say that indignant has stress on the second syllable or that the word like is stressed in Does 
he like it?" (60) Se puede, pues, observar cómo existe una fusión o confusión entre las dos partes que en español aparecen tan claramente contrapuestas, como ya se ha visto en los comentarios del libro anteriormente citado. Aunque en inglés existan, evidentemente, acentos dentro de la palabra, el modo en el que los golpes de voz se cuentan a la hora de un análisis entonacional, se basa más en los acentos encontrados en la secuencia de producción, como partes integrantes del todo, que en los casos aislados de la sílaba. Ésta es la razón por la que los alumnos cuya lengua materna es el inglés encuentran una dificultad especial en el momento de emplear las reglas de acentuación gráfica (y, por supuesto, prosódica) en este idioma.

J.F. Kaplan, en su estudio English Grammar: Principles and Facts, considera al tratar la estructura de las palabras inglesas la división de las mismas desde un punto de vista morfosemántico, es decir, basándose en los morfemas (71). Tras introducir este común modo de tratar la palabra, parece hacer un especial énfasis en las diferencias entre estos componentes y las propias sílabas: "A morpheme can be a single syllable, or several syllables long. It can even be less than a syllable, like the morpheme -s." (146) En el caso que nos ocupa se observa que, en numerosas ocasiones, los alumnos tienden a entender una potencial ruptura de la palabra en su concepto morfológico, y a partir de la misma una acentuación que a veces puede coincidir con la forma correcta, pero que está basada en una metodología disfuncional.

Así, como se podrá observar de una manera más detenida en el momento de analizar la práctica que los alumnos de este curso llevaron a cabo ya en una fase final de su aprendizaje, hay una serie de rasgos fonológicos del sistema lingüístico inglés que están interfiriendo de manera determinante en la asimilación y producción del sistema español en estos estudiantes: diferente estructura silábica ("clusters"); debilitamiento de vocales interconsonánticas en inglés, etc.

\section{HIPÓTESIS Y MÉTODO}

Los estudiantes de la clase de español SPN 111 (primer curso de enseñanza de la lengua) se hallan ante la dificultad de colocar la tilde sobre la letra que corresponde en la palabra de acuerdo con las reglas ortográficas del español. Tras diversas intervenciones, se observa que la raíz del problema parece hallarse en su dificultad para internalizar el sentido del concepto de sílaba en español, así como su forma. La causa que propicia este desajuste se encuentra en el proceso de interlenguaje con el que los alumnos tienden a fabricar su propia regulación lingüística, basándose en los golpes de voz que descubren en los vocablos a tratar. El que esos golpes de voz se hagan desde una mentalidad inmersa en su lengua materna, provoca interferencias insalvables que les conducen a interpretar erróneamente la L2.

No es posible para ellos decodificar las reglas de acentuación propuestas por la gramática española y basadas en la división silábica y esto produce a su vez un riesgo de fosilización que pueda causarles problemas en un estudio posterior de la acentuación gráfica en cursos superiores.

La clase de español en la que este estudio ha tenido lugar se enmarca en, como ya se ha adelantado, el estado de Nueva York y en la universidad pública SUNY de Stony Brook. Las particularidades de estos datos residen, primero, en el hecho de que sea una universidad pública a la que, en teoría, asiste gente de cualquier clase social, 
lo que la dota con un carácter heterogéneo ausente en muchos otros lugares. Si a esto se añade la información que se ha referido con anterioridad con respecto a la propia heterogeneidad cultural y racial que este lugar implica, se adquiere como resultado un grupo muy interesante por su diversidad, en el que es incluso difícil crear grupúsculos analizables con un mínimo de rigor que tengan muchos rasgos en común.

Se trata de un grupo de nivel básico SPN 111 y la sección con la que se identifican en el Department of Hispanic Languages and Literatures al que pertenecen es la 02. Hay veinticinco alumnos matriculados en este curso. Existe una división por géneros equitativa: once mujeres y once hombres. Existe entre ellos una gran variedad racial, social y cultural. Si hay algo que define a este grupo es, desde luego, la heterogeneidad. Está conformado por recién estrenados universitarios de diecisiete años y por mujeres que sobrepasan los cuarenta y cinco, que están casadas y que tienen hijos mayores. También de diferentes capas sociales y de muy distintos orígenes culturales, prácticamente la totalidad de los alumnos están perfectamente integrados en el grupo, y juntos han hecho que exista una gran dosis de confianza, tanto entre ellos mismos como entre ellos y el propio proceso de aprendizaje.

Lo que resulta especialmente relevante es la diferenciación entre las varias procedencias de este grupo, ya que es un dato que afecta directamente a la competencia lingüística de los estudiantes. Resulta curioso que los mejores estudiantes sean personas que han estado anteriormente expuestas al aprendizaje de una segunda lengua, aun a pesar de que no sea ésta el español. Una alumna francesa, otra de origen del mismo país europeo, un estudiante eslovaco que está en la clase en condición de oyente, un alumno de origen y competencia lingüística hebrea aceptable, una coreana y gente que lleva al menos cinco años estudiando otra lengua. Este subgrupo representa la "masa social" de los que mejores resultados están consiguiendo en esta clase, condición que se puede dar por dos razones: en primer lugar, porque las lenguas que estas personas tienen como L1 sean más próximas al español que el inglés. Esta posibilidad se debilita con la inclusión del coreano y del hebreo en la estadística, ya que aunque el francés es una lengua directamente emparentada con el español, y el eslovaco a su vez comparte una pronunciación relativamente similar a la de la lengua cervantina, no se puede decir lo mismo de las otras dos lenguas que hacen presencia a modo de interlenguaje en el grupo escogido para este estudio. La segunda -y más probableopción se fundamenta en la concepción de que la exposición a un entorno bilingüe capacita a la mente humana a adquirir una habilidad lingüística que los individuos que han crecido en una sociedad o en un ambiente monolingüe no poseen. Estas dos posibilidades - que no pueden ser consideradas como excluyentes o absolutas- precipitan un estado de recepción más inmediato y más analítico.

La realidad cotidiana de este alumnado permitió iniciar esta investigación a partir de una serie de explicaciones tuteladas que tuvieron lugar durante las primeras semanas del curso académico. Los principales problemas entre el alumnado (incluso el más avanzado) estaban, desde luego, relacionados con la entonación de las palabras. A pesar de que el alumnado comprendía perfectamente las reglas expuestas en sus libros de texto, a la hora de realizar ejercicios prácticos fallaban claramente. La observación de sus reacciones ante la comprensión de las reglas de acentuación y su posterior fracaso práctico llevó a considerar que el problema se hallaba en explicar las reglas ortográficas de acentuación en relación con la posición de la sílaba combinada y con el golpe de voz sobre ella. Los alumnos no fueron capaces de aislar la correcta formación de la 
sílaba en lengua española. A raíz de este problema, la atención se centró en el modo de actuación del alumnado con respecto al énfasis fónico, y en concreto, en el ámbito de la escritura como reflejo de su concepción fonológica.

\section{Desarrollo Del ANÁLISIS}

Con el fin de explicar y justificar la teoría que propongo en torno a la necesidad de una explicación teórica sobre la silabificación española que sirva de referente y guía para un posterior entendimiento de las reglas de acentuación, he seguido un proceso de trabajo individual tras el que los alumnos se vieron muy favorecidos para llevar a cabo de una manera exitosa este aspecto de su aprendizaje. Una actividad concreta con el alumnado sobre acentuación permitió observar una clara mejoría en la identificación acentual, ya que en este caso las palabras seleccionadas mostraban la sílaba tónica en negrita, por lo que reconocer dónde recaía el acento prosódico fue una tarea mucho menos problemática para ellos. Aun así, se puede observar una importante presencia de errores en la actividad a la hora de colocar el acento gráfico, probablemente debidos a una falta de estudio de las reglas gramaticales por un lado, o por otro, a que la hipótesis aquí planteada se justifique mediante la aparición de errores en los ejercicios del alumnado por una mala interpretación de la sílaba en lengua española. El método utilizado para llegar a tal reflexión fue el de integrar entre la serie de palabras expuestas al alumnado tres que eran de acentuación esdrújula, con el fin de aislar los casos de errores debidos a una falta de estudio por parte del alumnado, y poder así centrar el análisis en los casos que, conociendo las reglas de acentuación, fallan a la hora de colocar correctamente las tildes correspondientes. El resultado de tal experimento, sin embargo, es sorprendente, ya que se dan casos de personas que tratan de manera perfectamente correcta las palabras agudas, por lo que parecen conocer las reglas de acentuación, pero fallan luego muy claramente con la serie de palabras esdrújulas. Estas palabras esdrújulas comparten una estructura muy similar: $\mathrm{CV}-\mathrm{CV}-\mathrm{CV}(\mathrm{C}=$ consonante; $\mathrm{V}=$ vocal $)$, lo que parece arrojar que existe una tendencia entre el alumnado cuya primera lengua es el inglés a omitir la vocal interconsonántica de la segunda sílaba en palabras esdrújulas de lengua española, convirtiéndolas en bisilábicas y, por lo tanto, en graves y sin acento. El vocablo "vértice", por ejemplo, sería leído por estos alumnos como una palabra bisílaba compuesta por "vert-" y “-ce" causada por la debilidad de la "-i-" de la segunda sílaba, de acuerdo con los parámetros de pronunciación en lengua inglesa. Esta técnica, que se repetirá a lo largo de varios de los ejemplos que aparecen en el ejercicio práctico que he propuesto para este comentario, es una aplicación al español de la consideración de Kaplan de que "...English spelling is inadequate as a consistent representation of sounds." (68) Es decir, no existe una severa relación entre lo que se pronuncia y lo que se escribe como sucede en español. Esta "dificultad" a la que los estudiantes de SPN 111 están acostumbrados, se convierte aquí en un problema cuando los mismos, a través de un proceso de interlenguaje, inconscientemente adaptan ese rasgo de su lengua a la que están aprendiendo.

Resulta necesario explicar que la interacción entre el código escrito y el código oral pivota sustancialmente en los planteamientos de este estudio. Es precisamente la problemática que se establece con la separación de uno y otro con lo que este artículo 
baraja diferentes posibilidades de actuación. Las tildes, la separación silábica, o la acentuación pertenecen a esa tierra de nadie (o tierra de todos) en la que la variación de uno de los códigos tiene un impacto directo sobre el otro, y es en esa fluctuación de poderes en la que se mueve este estudio. En 2002, Emilia Ferreiro plantea una cuestión similar:

La irreductibilidad de la escritura a la noción de código ya ha sido materia de reflexión (Blanche-Benaviste). Si fuera una simple codificación, las unidades de análisis de lo oral deberían encontrarse en la escritura, y no es el caso. No hay correspondencia unívoca entre letras y fonemas (en las diferentes escrituras alfabéticas hay poligrafías para un mismo fonema y polifonía para un mismo grafema). No hay correspondencias unívocas entre las segmentaciones de lo escrito -las palabras gráficas- y los morfemas. La mayúscula y el punto segmentan oraciones, una entidad que sólo tiene realidad en la escritura, según varios lingüistas contemporáneos (Beguelin). (Ferreiro, 152)

Las injerencias de lo oral sobre lo escrito y de lo escrito sobre lo oral se manifiestan en la acentuación de una forma muy explícita, y es esa forma la que interesa en este estudio. Los casos estudiados demuestran una clara intromisión de la forma en la que -por medio de una evidente fosilización- pronuncian las palabras en L2 siguiendo los parámetros de pronunciación establecidos por su L1, y plasman tal desajuste en su resolución al ejercicio en la representación gráfica de la separación silábica.

La siguiente lista de palabras responde a una actividad asignada a este mismo grupo de estudiantes con el fin de que ejercitasen la separación silábica y, posteriormente, acentuasen las palabras donde fuese necesario. Las conclusiones a las que condujeron las repuestas de los alumnos aparecen reflejadas al término de la exposición del ejercicio, y parecen afirmar la problemática condición de la transferencia lingüística en referencia a la acentuación gráfica. Tal y como se explicó con anterioridad, resulta interesante observar que la naturaleza heterogénea -desde un punto de vista étnico, cultural y, sobre todo, lingüístico- característica de una clase de español como segunda lengua en una universidad neoyorquina permite que la muestra analizada, el estudio de caso propuesto, permita observar las injerencias de las diferentes L1 en la división silábica en español. Aunque no es ésa la función principal de este estudio -que, recordemos, busca indagar en la afirmación de Elaine Tarone de que el origen de los problemas del alumnado cuya L1 es el inglés para colocar acentos gráficos radica no tanto en la falta de acierto a la hora de localizar la sílaba tónica de la palabra, sino en el propio mecanismo de identificación de las sílabas y en la problemática división de palabras en sílabas- entendemos que la influencia de otras lenguas en el aprendizaje del español, aun cuando la lengua vehicular entre los casos analizados es el inglés, puede repercutir en las dificultades mostradas. Sin duda un campo de investigación tremendamente atractivo, pero que en este estudio se tratará sólo de manera tangencial por escapar a las competencias de las líneas de estudio propuestas en él.

\section{ACTIVIDAD PRÁCTICA DE DIVISIÓN SILÁBICA Y ACENTUACIÓN GRÁFICA}

Dado que el temario de SPN 111 no pone un especial énfasis en la importancia de la acentuación prosódica o gráfica, resulta un tanto difícil recoger un corpus con datos 
que sean realmente fiables, ya que el alumno ha de presentarse voluntariamente a la realización de un ejercicio de estas características, y eso en sí puede suponer una perversión de la objetividad del estudio, ya que responden a un determinado perfil los alumnos que se muestran interesados en colaborar con este tipo de experimentación.

Por eso, en sólo una actividad ha sido efectiva la colaboración desinteresada de la totalidad del grupo, que se ha mostrado dispuesto a colaborar tras conocer los objetivos del estudio. Esta actividad consiste directamente en separar en sílabas una serie de palabras entre las que se encuentran algunas conocidas para ellos, pero otras completamente nuevas. Curiosamente, éste no fue un factor que determinó la corrección o incorrección de los resultados de la actividad. En general, estos resultados han aportado una información que parece dirigir el grado de corrección hacia cuán próxima la palabra que están tratando de analizar se encuentra de su propia lengua, que asumimos es, en rasgos generales, el inglés (y teniendo en cuenta, como decíamos, la influencia de otras lenguas en casos de alumnos de nacionalidad u origen lingüístico diverso).

Las palabras propuestas para este ejercicio práctico responden a una acumulación de factores silábicos en cada una de ellas que pueden llevar al sujeto participante en el estudio a aplicar elementos de su propia fosilización. Si bien el corpus citado explícitamente no es lo suficientemente extenso como para probar fehacientemente la hipótesis formulada en este estudio, sí es representativo de una aproximación inicial a la influencia de las dificultades silábicas de los participantes sobre su producción lingüística, que es precisamente el objetivo de este artículo. El ejercicio en sí, al ser realizado a lo largo de un semestre académico, implicó el análisis de varias decenas de palabras, de las cuales se han recogido para este estudio las que resumen las conclusiones generales del análisis (grupos consonánticos determinados, diptongos e hiatos, etc). Se omite, sin embargo, el análisis de los casos que pudiesen resultar redundantes o que no conllevarían una carga semántica adicional para los objetivos científicos de este estudio.

Asimismo, la configuración de este listado adquiere gran valor en este sentido, al contar con la participación de un grupo extenso y heterogéneo de sujetos hablantes que se enfrenta a las dificultades de cada caso de una forma diferente pero convergente, lo que permite observar ciertas tendencias comunes.

Las palabras elegidas para evidenciar las conclusiones de este estudio son las siguientes (obsérvese en letra cursiva la explicación de la dificultad que conlleva cada una):

\footnotetext{
"Aburrido": Con esta palabra, el principal problema se da con la doble consonante, que se tiende a separar. En los casos en los que los alumnos las colocan juntas, lo hacen dentro de la sílaba "-bu-", dejando la "-i-" sola. Esto se puede deber a la dificultad que presenta la sílaba inglesa para albergar el patrón CV. También se puede deber a la propia dificultad del sonido vibrante y líquido (r), que es, sin embargo, más fácilmente pronunciable al final de la sílaba que al principio de la misma.

"Cansado": Esta palabra no ofreció problemas. Sólo en algunos casos sucedió que fue dividida en dos sílabas en lugar de en tres. La posible dificultad de este ejemplo residía en la colocación agrupada del conjunto consonántico -ns-por un lado, y la presencia de la terminación -ado, que no obstante no pareció afectar el análisis de los participantes. "Aburrimiento": Igual que "aburrido", pero al mismo tiempo con fallos también en la penúltima sílaba [mien], que aparece separada. No es un error grave a este nivel, pero
} 
da pistas de cómo puede haber confusión entre a la hora de contabilizar las sílabas para acentuar, entre los alumnos cuya L1 es el inglés.

"Fertilidad": Un buen ejemplo de la confusión silábica; existen grandes problemas con las sílabas interiores: "tili", "til-i", "ti-lid". Este error deberse a la rapidez de producción oral del inglés con respecto al español, producida por un debilitamiento de la vocal interconsonántica (que tiende a convertirse en una vocal neutra, o simplemente a desaparecer), y cuya fuerza en español se mantiene.

"Fanatismo": Un 60\% de los estudiantes tienden a separar esta palabra de acuerdo a su propio conocimiento dentro de la L1. Usan "fan" como una sola sílaba. Se trata de un claro ejemplo de interlenguaje.

"Versatil": Es éste un caso curioso, ya que a pesar de haber sido bien dividido en la mayoría de los casos, quienes pusieron acento, lo hicieron en la última sílaba, lo que es contrario a la norma. Esto pudo deberse a que no recibieron instrucción de colocar el acento gráfico hasta que hubieron terminado de hacer la separación silábica, y en ese momento ya se encontraban desorientados ante la multitud de ejemplos como para poder intentar aplicar las reglas ortográficas. Es un dato importante, ya que apoya la hipótesis de este artículo en torno a la necesidad de una explicación más rigurosa de la sílaba en español, previa a la explicación de las reglas de acentuación en esta lengua.

"Sincronia": El 93\% de los resultados no presentó importantes problemas, más que con las dos últimas sílabas. Entre ellos, el $45 \%$ las trató como dos diferentes, y el 55\% como sólo una. Hay, sin embargo, dos casos en los que se presenta un problema también con las primeras sílabas: sinc-ro y sin-cron.

"Desfallecer": Palabra muy problemática. Los estudiantes ven su tarea dificultada por las dobles consonantes y los grupos consonánticos, lo que se traduce en terminaciones incorrectas a la hora de separar la palabra. Es observable especialmente que no hay un concepto claro de lo que es una sílaba.

"Atraccion": Hay resultados más dispares. Hay quien considera "a" como una sílaba y quienes la mantienen pegada a la t: "at-". Es quizá la palabra más complicada, ya que ha habido numerosas soluciones diferentes. La dificultad de esta palabra es extrema, ya que incluso los hablantes nativos de la lengua (en un foro diferente) no fueron capaces de realizar con éxito y corrección la división silábica, por lo que su presencia en esta lista estaba justificada como culmen de la dificultad práctica.

"Lampara": Como se preveía, gran tendencia a hacer de esta palabra sólo dos sílabas: lam-para. Desde luego, tiene que ver el debilitamiento de la pronunciación vocálica de la " $a$ " inglesa en posición interconsonántica para entender este error. Quirk lo expresa mediante el ejemplo de la palabra en lengua inglesa "words", cuya transcripción fonética resulta en la desaparición de la vocal equivalente a la del ejemplo expuesto en este ejercicio: [wrdz] (Quirk, 78).

"Rectangulo": Bien dividida en general, aunque hay un $18 \%$ de respuestas que empieza la división con "rect-", de la misma manera que hay un 14\% que deja el conjunto "-gulo" sin separar. De nuevo creo que se debe a un ejemplo de interlenguaje, donde la vocal no existe en inglés, y su pronunciación en español se adivina mucho más débil.

"Clavija": Con mucha más unidad, sólo se da el caso de una persona que considera “-vija” como una sola sílaba, y dos personas que incluyen la “-v-” en la primera sílaba. La pronunciación aplicada por los voluntarios que cometen este error probablemente sea /dz/.

"Salud inestable": Sólo se ha dado un caso en el que se consideran estas dos palabras dentro de la misma división silábica [sal-ud-in-est-able], y otros dos casos en los que se establece la misma sinalefa aunque con una división silábica diferente. Es una representación de la tendencia de la lengua inglesa oral a formular dicciones conjuntas de palabras diferentes: "hit her" [' $h$ i itr]. 
Los trabajos originales reflejan varios puntos importantes que han de ser comentados. El primero de ellos es que existe una gran variedad en el rango de respuestas que los alumnos dieron, por lo que se entiende que la táctica que se ha seguido en el libro de texto utilizado en la clase para introducir esta nueva información ha sido, cuando menos, poco productiva. Resulta curioso atender al hecho de que aquellos alumnos que se interesaron por mejorar este aspecto de su aprendizaje y que recibieron así una mejor (o mayor) formación en cuanto a la sílaba, obtuvieron unos resultados en esta actividad mucho más logrados. Quizá se debió a que precisamente estaban mucho más motivados, pero también puede ser que simplemente necesitan una mayor profundización en su conocimiento silábico. Se encuentran problemas también en la conformación de los patrones silábicos en español, sobre todo en los momentos en los que aparecen dos o más consonantes juntas y en los que se tiende a utilizar más el grupo CVC que el CV que impera en el sistema silábico de la lengua cervantina. Además de este rasgo y del anteriormente comentado que trata del debilitamiento de la vocal interconsonántica en palabras esdrújulas y, de manera menos incipiente, en las graves ("computadora"), hay que hablar, asimismo, del problema de la división morfemática. Se dan casos en los que los alumnos tienden a identificar la sílaba con los morfemas componenciales de la palabra y, así, adquieren resultados del tipo de: "fanatismo"= "fan-at-ism-o", donde el alumno ha aislado los morfemas que reconoce y ha dejado el resto de la palabra a su libre criterio. Otro caso interesante lo representa el sintagma nominal "salud inestable" ya que, como se comentaba con anterioridad, hubo tres casos en que la separación silábica se realizó como una sola unidad. Esto es muy indicativo para reflexionar sobre cómo en inglés la continuidad entonacional trasciende los límites de la propia palabra y cómo los alumnos se dejan arrastrar por la marea del interlenguaje hacia el error ortográfico.

Además de los datos intrínsecamente relacionados con el motivo de esta investigación, el ejercicio propuesto ha permitido la recolección de otro tipo de datos que pueden ser útiles en las discusiones venideras sobre el tema tratado. Datos de interés que se recogen en los resultados de las intervenciones por parte de los estudiantes son los que ofrecen quienes no son capaces de aislar el concepto de "tilde". Son conscientes de que existe algo que es en ocasiones utilizado, pero que no pueden asimilar. Esto se nota en los ejemplos escritos, porque no es extraño encontrarse con alumnos que escriben la palabra "tíos" con tilde en la "t-". En este mismo apartado también se incluyen aquellos alumnos que escriben toda palabra grave con tilde, sea cual sea su terminación, en lo que podría interpretarse como un vencimiento ante la frustración de no disponer de las herramientas de conocimiento suficientes para operar adecuadamente en el ámbito de la acentuación gráfica y de la separación en sílabas. Otra subcategoría podría ser la que incluye palabras perfectamente acentuadas a pesar de su dificultad silábica, debido a que son préstamos del inglés y su pronunciación es idéntica a la de esta lengua: fútbol, suéter, etc. Un grupo de palabras muy interesante es el que tiene como características más importantes o bien la supresión o bien la inclusión de letras dentro de la propia palabra, creando una serie de problemas en el apartado de acentuación. La supresión o inclusión de letras en una palabra puede provocar su transformación silábica y la consiguiente manipulación errónea de las reglas de acentuación gráfica. Ejemplos de este caso son: "veintun"; "complenos" (cumpleaños); "esculas"; "incluiuyendo"; "nevo" (nuevo); "clonias” (colonias); "celbrasen” (celebrasen), etc. Con estos casos, se produce una plasmación gráfica del 
proceso fónico desarrollado por los estudiantes y que es tan propicio en el ámbito del interlenguaje: la asimilación de las palabras de la L2 por medio de un proceso de fosilización como variantes de la grafía -o sonido- de la versión de esa misma palabra que ellos tienen internalizada en la L1.

Existe, pues, una serie de causas muy divergentes entre sí, pero con el rasgo común de presentar problemas debidos a la sílaba y al interlenguaje, para tratar de explicar las dificultades que se han venido tratando a lo largo de este trabajo. Según la hipótesis aquí desarrollada, es muy probable que muchos de estos problemas sean resueltos con una explicación teórica a los alumnos sobre la sílaba en lengua española previa a la introducción de los acentos y sus reglas gramaticales. No se han de tratar, desde luego, como instancias separadas, y mucho menos ha de supeditarse la una a la otra de forma jerárquica, ya que resulta evidente que los alumnos responden positivamente al manejar ambas temáticas simultáneamente.

\section{CONCLUSIONES}

Coincidiendo con los dos principales textos en los que se ha basado esta investigación (Tarone y Greenberg), los resultados de la misma parecen arrojar que la estructura silábica del interlenguaje es con frecuencia diferente a la de la L2, y que el proceso dominante que influye en la estructura silábica de la fonología del interlenguaje, resulta ser un problema de transferencia lingüística.

Es especialmente recurrente entre los alumnos de un nivel tan básico como este, tener que acudir a su lengua materna para recrear la interpretación de referentes en un significante global en relación hiponímica con el resto de lenguas. En este viaje de una lengua a otra aparecen numerosas incorrecciones debido a la condición única de cada lengua y a la imposibilidad de transferencia exacta desde un código hacia el otro y la consecuente creación de traumas lingüísticos. Se trata de un proceso de pre-fosilización que debería ser atajado de inmediato.

Sin embargo, no creo que este trabajo aporte conclusiones definitivas a un problema de este tipo. Está en realidad concebido como forma de apertura para una posterior investigación de un campo que no ha sido ampliamente tratado y que, por su relevancia como base para el mejor entendimiento de la adquisición de una L2, sería de extremo interés para futuros estudios sobre este campo.

\section{OBRAS CITADAS}

Abercrombie, David. 1981. Studies in Phonetics and Linguistics. Oxford: Oxford University Press.

Alarcos Llorach, Emilio. 1994. Gramática de la Lengua Española. Madrid: Espasa Calpe.

Aragó, M. R. 1995. Diccionario de dudas y problemas del idioma español. Buenos Aires: El Ateneo.

Caycedo Garner, Lucía et al. 2001. ¡Claro que sí! Fifth Edition. Orlando: Houghton Mifflin Harcourt (HMH)

Corder, S.P. 1981. Error Analysis and Interlanguage. Oxford: Oxford University Press. 
Ferreiro, Emilia. 2002. "Escritura y oralidad: unidades, niveles de análisis y conciencia metalingüística”. En E. Ferreiro (Ed.), Relaciones de (in)dependencia entre oralidad y escritura. Barcelona: Geddisa Editorial.

Grabe, W. y Kaplan, R. 1991. Introduction to Applied Linguistics. New York: Addison-Wesley Publishing Company.

Greenberg, Cindy. 1983. "Syllable Structure in Second Language Acquisition". CUNYforum, 9. 41-65 (Queens College and The Graduate Center, CUNY).

Hodne, Barbara. 2006. "Yet Another Look at Interlanguage Phonology: The Modification of English Syllable Structure by Native Speakers of Polish". Language Learning, vol. 35.3 (405-417).

Kaplan, Jeffrey P. 1989. English Grammar: Principles and Facts. New Jersey: Prentice Hall.

Krashen, Stephen D. 1981. Second Language Acquisition and Second Language Learning. Oxford: Pergamon Institute of English.

McCarthy, J. 1979. “On Stress and Syllabification”. Linguistic Inquiry, 10.3. 443-465.

Nehls, Dietrich, ed. 1987. Interlanguage Studies. Gross: Heidelberg.

Quirk, Randolph, 1973. A Concise Grammar of Contemporary English. London: Harcourt Brace Jovanovich, Inc.

Roulet, Eddy. 1971. Linguistic Theory, Linguistic description and Language Teaching. London: Longman.

Selinker, Larry. 1992. Rediscovering Interlanguage. Applied Linguistics and Language Study. Michigan: Longman.

Sato, Charlene J. 2006. "Phonological processes in second language acquisition: another look at interlanguage syllable structure". Language Learning, vol. 34.4 (43-58).

Tarone, Elaine. 1980. "Some influences on the Syllable Structure of Interlanguage Phonology". International Review of Applied Linguistics in Language Teaching, 18.2 139-52. - 1998. "Interlanguage". K. Johnson y H. Johnson, eds. Encyclopedic Dictionary of Applied Linguistics: A Handbook for Language Teaching. Massachusetts: Blackwell. 507-512.

Van Els, Theo et al. 1984. Applied Linguistics and the Learning and Teaching of Foreign Language. London: Wolters-Noordhoff. 